\title{
A necessidade da imunofluorescência direta no diagnóstico da dermatose bolhosa por IgA
}

Primeira submissão em 10/07/11 Última submissão em 27/11/11 Aceito para publicação em 29/11/11 Publicado em 20/02/12

The need for direct immunofluorescence in the diagnosis of IgA bullous dermatosis

Daniel Chang

unitermos

Dermatose bolhosa

Doença autoimune

Imunofluorescência direta

\section{resumo}

A dermatose bolhosa por imunoglobulina da classe A linear (DblgA) do adulto é uma doença autoimune rara caracterizada por formação de bolhas subepidérmicas e depósito linear de imunoglobulina da classe A ( $\lg A)$ na zona da membrana basal (ZMB). Por possuir aspectos clínicos e histológicos semelhantes a outras dermatoses bolhosas, principalmente a dermatite herpetiforme e o penfigoide bolhoso, faz-se necessária a realização de imunofluorescência direta para confirmação diagnóstica. Apresenta-se então, neste artigo, relato de caso ilustrando essa necessidade. abstract

Linear immunoglobulin A bullous dermatosis (DblgA) of adults is a rare autoimmune disease characterized by subepidermal blistering and linear deposits of immunoglobulin $A(\lg A)$ in the basement membrane zone (BMZ). Owing to the fact it presents clinical and histological aspects similar to other bullous dermatosis, mainly dermatitis herpetiformis and bullous pemphigoid, direct immunofluorescence is required to confirm diagnosis. In this article, we describe a case that illustrates this need. key words

Bullous dermatosis

Autoimmune disease

Direct

immunofluorescence 


\section{Introdução}

A dermatose bolhosa por imunoglobulina da classe A linear (DblgA) representa uma doença bolhosa autoimune rara que se caracteriza pela formação de bolhas com nível de clivagem subepidérmica e deposição de anticorpo do tipo imunoglobulina da classe $\mathrm{A}(\lg \mathrm{A})$ de padrão linear e homogêneo na zona da membrana basal $(\mathrm{ZMB})^{(3)}$. Clínica e histopatologicamente, é muito similar a outras dermatoses bolhosas, sobretudo a dermatite herpetiforme (DH) e o penfigoide bolhoso (PB). Assim, para diagnóstico preciso, faz-se necessária a realização de imunofluorescência direta $(\text { IFD })^{(1,9)}$. Apresenta-se, a seguir, relato de caso ilustrando tal exigência.

\section{Relato do caso}

Adulto feminino, 21 anos, exibindo lesões vesicobolhosas tensas com conteúdo claro, agrupadas e sob base eritematosa com leve prurido e ardor, localizadas no tronco e na mucosa oral. Não havia relatos de uso de medicações e nem outras doenças associadas. Realizaram-se as biópsias da lesão para avaliação histológica e da área perilesional para IFD. A análise histopatológica exibia clivagens subepidérmicas permeadas por infiltrado inflamatório polimorfonuclear composto por frequentes neutrófilos e alguns eosinófilos (Figura 1), além de microabscessos neutrofílicos em papilas dérmicas (Figura 2). Realizou-se, também, o painel de IFD com pesquisa de anticorpos IgA (anti-human IgA, alfa chain specific, Dako), imunoglobulina da classe $\mathrm{G}(\lg \mathrm{G})$ (anti-human $\lg \mathrm{G}$, whole molecule, Dako), imunoglobulina da classe M (IgM) (anti-human IgM, $\mu$ chain, Dako), C3c (complement, Dako) e fibrinogênio (anti-fibrinogen, Dako) resultando em positividade apenas para IgA com padrão linear e homogêneo em ZMB, confirmando o diagnóstico de DblgA do adulto (Figura 3). A imunofluorescência indireta resultou negativa. Após, foi instituído tratamento medicamentoso com corticoterapia sistêmica com melhora significativa das lesões.

\section{Discussão}

A DblgA, descrita em 1901 por Bowen, é uma dermatose bolhosa autoimune rara com clivagem subepidérmica do ponto de vista histopatológico e se caracteriza pela presença de depósito linear e homogêneo de anticorpo $\lg \mathrm{A}$ em $\mathrm{ZMB}^{(4)}$.

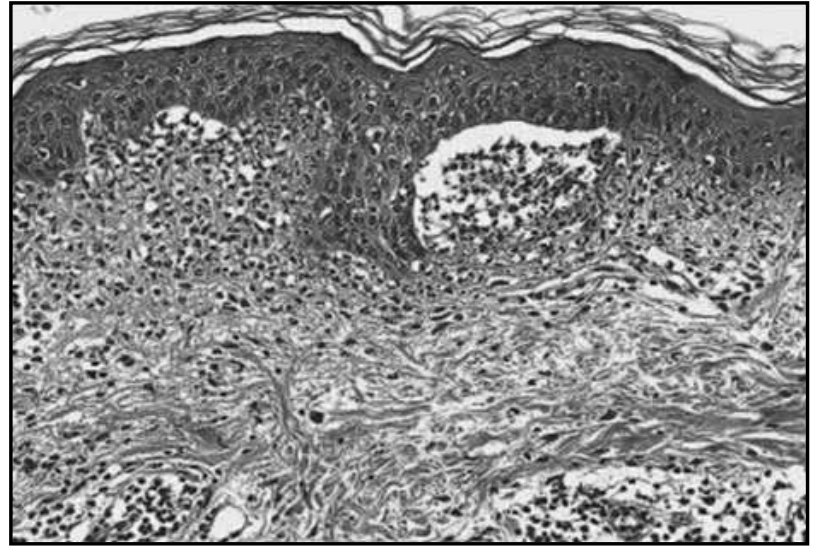

Figura 1 - Clivagem subepidérmica com infiltrado inflamatório polimorfonuclear com neutrófilos e eosinófilos (HE, aumento de 100x)

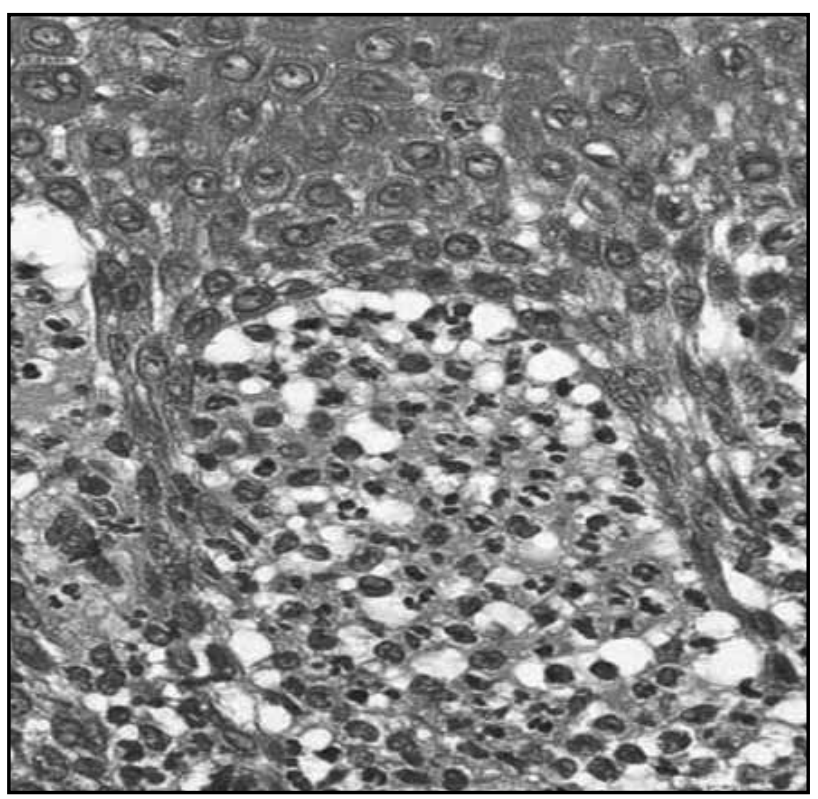

Figura 2 - Microabscessos em papila dérmica (HE, aumento de 400x)

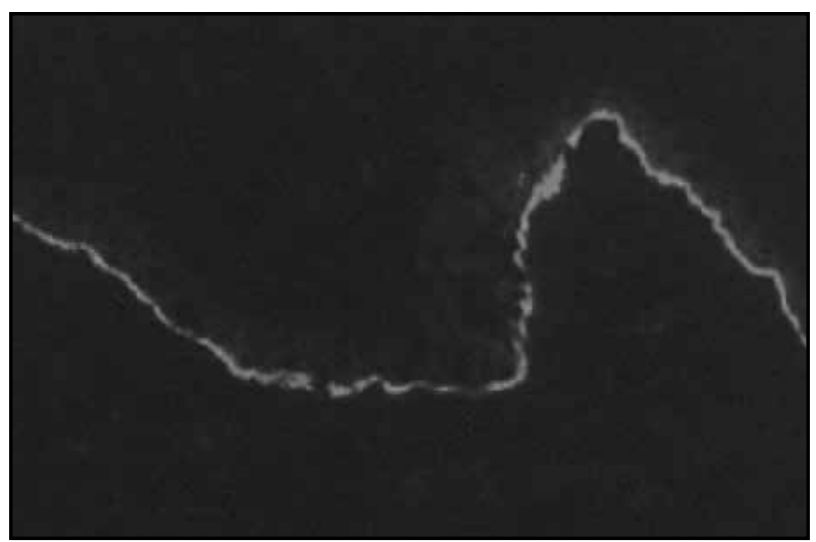

Figura 3 - Imunofluorescência direta. Depósito linear e homogêneo de IgA em ZMB (aumento de 100x)

IgA: imunoglobulina da classe A; ZMB: zona de membrana basal.

Há duas variantes clínicas: da infância e do adulto. A primeira, também denominada dermatose bolhosa crônica da infância, é mais comum entre 2 e 5 anos de idade e 
acomete preferencialmente a região perineal, o abdome inferior e a região perioral, raramente havendo o envolvimento de mucosas. Na forma adulta, que surge após a puberdade, o quadro clínico assemelha-se à $\mathrm{DH}$ ou ao $\mathrm{PB}$ e o acometimento mucoso é mais comum ${ }^{(2)}$.

Há evidências na literatura de associação, em alguns casos, do surgimento de DblgA ao uso de medicamentos ${ }^{(10)}$.

O exame histopatológico evidencia a clivagem subepidérmica com infiltrado inflamatório rico em polimorfonucleares, principalmente neutrófilos, mas contendo, também, eosinófilos. Microabscessos em papilas dérmicas podem, ainda, ser encontrados ${ }^{(1,9)}$. Pelo fato de outras dermatoses bolhosas autoimunes, principalmente a DH e o PB, cursarem com os mesmos achados histológicos, torna-se peremptório a realização de IFD para confirmação diagnóstica.

Em relação à IFD, os principais alvos dos autoanticorpos são domínios extracelulares de $120 \mathrm{kDa}$ (LAD-1) e de 97 kDa (LABD-97) do BP 180 (colágeno XVII) e é descrito, ainda, con- tra colágeno VII, BP 230, alfa6beta4 integrina, laminina, entre outras $^{(6,15,16)}$. Quanto aos títulos de lgA circulante, constatam-se, aproximadamente, $60 \%$ a $70 \%$ de IFD positivas ${ }^{(4)}$.

Em até $25 \%$ dos casos, pode-se demonstrar também a presença de anticorpo $\lg G$, juntamente com IgA, na ZMB nas DblgA, fornecendo evidências de um subtipo de DblgA com resposta autoimune dual de lgA e lg $\mathrm{G}$ contra o $\mathrm{BP} 180^{(8,14)}$. Do ponto de vista clínico, não há diferenças entre os tipos apenas com marcação para IgA daqueles com expressão de lgA e lgG; este último contribui menos frequentemente para o tipo infantil em relação ao adulto(5).

Assim, IFD é o método laboratorial fundamental para o diagnóstico exato, já que somente dados clínicos e histopatológicos não são suficientemente sensíveis e específicos para o diagnóstico preciso das dermatoses bolhosas autoimunes. Dessa forma, a deposição linear e homogênea de IgA na ZMB confirma o diagnóstico de DblgA, haja vista que na $\mathrm{DH}$ o $\lg \mathrm{A}$ se deposita de forma granular em derme papilar, enquanto no PB há deposição linear e homogênea em ZMB de $C 3^{(7,11-13)}$.

\section{Referências}

1. BICKLE, K.; ROARK, T. R.; HSU, S. Autoimmune bullous dermatoses: a review. Am Fam Physician, v. 65, p. 1861-70, 2002.

2. BRAUN-FLACO, O. et al. Blistering diseases. In: BRAUNFALCO, O. et al. Dermatology. 2. ed. Milano: SpringerVerlag, 2000, p. 648-95.

3. EGAN, C. A.; ZONE, J. J. Linear IgA bullous dermatosis. Int J Dermatol, v. 38, p. 818-27, 1999.

4. GUIDE, S.V.; MARINKOVICH, M.P. Linear IgA bullous dermatosis. Clin Dermatol, v. 19, p. 719-27, 2001.

5. HORIGUCHI, Y. et al. Linear IgA dermatosis: report of an infantile case and analysis of 213 cases in Japan. J Dermatol, v. 35, p. 737-43, 2008.

6. HORVATH, B. et al. IgA autoantibodies in the pemphigoids and linear IgA bullous dermatosis. Exp Dermatol, v. 19, p. 648-53, 2010.

7. ICHARA, V. K.; RAJALAKSLMI, T. Direct immunofluorescence in cutaneous vesicobulloses lesions. Ind J Pathol Microbiol, v. 50, p. 730-2, 2007.

8. METZ, B. J. et al. Linear IgA dermatosis with IgA and IgG autoantibodies to the $180 \mathrm{kDa}$ bullous pemphigoid antigen (BP180): evidence for a distinct subtype. Int J Dermatol, v. 43, p. 443-6, 2004.

9. PATRICIO, P. et al. Autoimmunes bullous dermatosis: a review. Ann N Y Acad Sci, v. 1173, p. 203-10, 2009.

10. Plunkett, R.W.; ChiAREllo, S. E.; Beutner, E. H. Linear bullous dermatosis in one of two piroxicaninduced eruptions: a distinct direct immunofluorescence trend revealed by the literature. J Am Acad Dermatol, v. 45, p. 691-6, 2001.
11. POHLA-GUBO, G.; HINTNER, H. Direct and indirect immunofluorescence for the diagnosis of bullous autoimmune diseases. Dermatol Clin, v. 29, p. 36572, 2011

12. SMITH, E. P.; ZONE, J. J. Dermatitis herpetiformis and linear IgA bullous dermatosis. Dermatol Clin, v. 11, p. 511-26, 1993.

13. VAN, L. et al. Dermatitis herpetiformis: potential for confusion with linear IgA bullous dermatosis on direct immunofluorescence. Dermatol Online J, v. 14, p. 21, 2008.

14. WOJNAROWSKA, F. et al. Chronic bullous disease of childhood, childhood cicatricial pemphigoid and linear IgA disease of adults. A comparative study demonstrating clinical and immunopathological overlap. J Am Acad Dermatol, v. 19, p. 792-805, 1988.

15. ZAMBRUNO, G.; KANITAKIS, J. Linear IgA dermatosis with IgA antibodies to type VII collagen. Br J Dermatol, v. 135, p. 1004-5, 1996.

16. ZILLIKENS, D. BP 180 as the common autoantigen in blistering disease with different clinical phenotypes. Keio J Med, v. 51, p. 21-8, 2002.
Endereço para correspondência

Daniel Chang

Rua Frei Caneca, 1.119 - Consolação

CEP: $01307-003$ - São Paulo-SP 